\title{
Diferencias de género en jóvenes universitarios del Ecuador, de acuerdo con sus intereses y capacidades para emprender
}

\section{Gender differences in university students from Ecuador, according to their interests and abilities in entrepreneurship}

\author{
Valentina Ramos Ramos 1 https://orcid.org/0000-0001-7803-8461, \\ Christopher Páramo Suárez ${ }^{1}$ https://orcid.org/0000-0002-9067-0416 \\ ${ }^{1}$ Escuela Politécnica Nacional, Quito, Ecuador \\ valentina.ramosdepn.edu.ec, christopher.paramodepn.edu.ec
}

\section{(c) $(1) \Theta$}

Esta obra está bajo una licencia internacional

Creative Commons Atribución-NoComercial 4.0.

$\begin{array}{ll}\text { Enviado: } & 2021 / 11 / 22 \\ \text { Aceptado: } & 2021 / 12 / 06 \\ \text { Publicado: } & 2021 / 12 / 30\end{array}$

\section{Resumen}

El emprendimiento es una característica presente en esta generación, por tanto, estudiantes universitarios tienen el interés de crear su propio negocio y ser independientes. Sin embargo, el contexto y la existencia de un entorno propicio para innovar y emprender son esenciales para que exista interés de emprender. Igualmente, la formación es importante para desarrollar capacidades necesarias para tener éxito en el emprendimiento. Este estudio muestra la comparación entre hombres y mujeres sobre los intereses y capacidades de emprendimiento en dos grupos de jóvenes universitarios: el primero de miembros de AIESEC y el segundo de estudiantes de últimos semestres de la carrera de Ingeniería Empresarial. La recolección de información se realizó mediante cuestionarios que evalúan: empatía, valores, inteligencia emocional, proactividad, entre otras competencias; además del interés para emprender. Los resultados muestran que hay diferencias de género en el caso de miembros de AIESEC en comparación con los estudiantes de administración. Donde, el género masculino tiene diferencias estadísticamente significativas en la percepción de excelencia para crear una empresa y la existencia de negocios previos en ambos grupos. Las personas de género femenino, por su parte, mostraron tener autoestima más baja, pero mayor apoyo del círculo cercano al momento de crear una empresa.

Palabras clave: capacidades, emprender, estudiantes universitarios, género, intereses.

Sumario: Introducción, Metodología, Resultados, Discusión y Conclusiones.

Como citar: Ramos, V. \& Páramo, C. (2021). Diferencias de género en jóvenes universitarios del Ecuador, de acuerdo con sus intereses y capacidades para emprender. Revista Tecnológica - Espol, 33(3), 23-39. http://www.rte.espol.edu.ec/index.php/tecnologica/article/view/885 


\begin{abstract}
Entrepreneurship is a characteristic of this current generation; therefore, university students are interested in creating their own business and being independent. However, the context and the existence of an environment conducive to innovation and entrepreneurship are essential to foster interest in entrepreneurship. Likewise, training is important to develop the necessary skills to succeed in entrepreneurship. This study shows the comparison between men and women on their interests and abilities of entrepreneurship in two groups of young university students: the first group of AIESEC (acronym in Spanish) members and the second group of students in their last semesters of Business Engineering. The information was collected through questionnaires that evaluate empathy, values, emotional intelligence, proactivity, among other competencies, as well as interest in entrepreneurship. The results show that there are gender differences in the case of AIESEC members compared to management students. Male gender has statistically significant differences in the perception of excellence to create a company and the existence of previous businesses in both groups. Females, on the other hand, showed lower self-esteem, but greater support from the close circle at the time of creating a business.
\end{abstract}

Keywords: entrepreneurship, gender, interests, university students, skills.

\title{
Introducción
}

La evolución de la sociedad se ha categorizado por la capacidad de adaptación e innovación del ser humano en el ecosistema que le rodea, es decir, la habilidades, conocimientos, capacidades e interés que posee le permiten mejorar su estilo de vida, sobre todo el factor económico. En base a esta premisa, en los últimos años las personas han buscado tener una independencia económica por medio del emprendimiento, poniendo en práctica sus propias capacidades e intereses que les permite crear un negocio propio y buscar su satisfacción personal. El emprendimiento se ha convertido en un elemento clave para el crecimiento de la economía de las naciones y una de las principales fuentes de empleo de los ciudadanos (Vuorio, Puumalainen \& Fellnhofer, 2018), determinándose como uno de los aspectos fundamentales la existencia de ecosistemas que permitan el surgimiento y desarrollo del emprendimiento (Malecki, 2018).

En la actualidad, el alto nivel de competitividad de las empresas tradicionales en la búsqueda de los mejores profesionales tanto en habilidades como en experiencia en el mercado laboral generan que el emprendimiento sea una opción cada vez más viable para las nuevas generaciones. Estas poseen capacidades que desarrollan en sus campos de estudio y la influencia de su red de apoyo que les permite transformar sus ideas para capitalizarlas en un negocio que se adapta de forma creativa e innovadora ante los frecuentes cambios y retos que se presentan en el mundo.

De esta forma se define al emprendedor como el individuo que crea un negocio en base a habilidades y conocimientos tanto aprendidos como adquiridos con el soporte de la influencia de su red de apoyo y su propia motivación para obtener un beneficio económico para ayudar a todo su entorno (Echeverri, 2018; Faghih, 2019; Ferreto, 2018; Krauss, 2018; Villalobos, 2018).

\section{Condiciones de emprendimiento}

Mediante el modelo de Paturel (1997) conocido como la coherencia del proyecto se determina que existen 3 factores base para emprender: motivaciones del creador, sus competencias y recursos. Además, que el ambiente donde se desarrolla la actividad emprendedora siempre busca ser favorable para su desarrollo. La intención de emprender no es un suceso al azar de la intencionalidad y bajo la influencia que recibe el futuro de su red de 
apoyo (Bird \& Jelinek, 1988). Para Gallurt-Plá (2010) el contexto social que está en contacto con el individuo le incentiva y afecta positivamente a la hora de crear un emprendimiento. Además, el círculo cercano que afecta positivamente a la persona que va a emprender, es decir, la familia y amigos son promotores para la emprender, ya que brindan apoyo y motivación constante (Iglesias, Jambrino, Peñafiel, \& Kokash, 2016).

\section{Capacidades de emprendimiento}

Los estudios sobre el emprendimiento muchas veces hacen referencias a los elementos relacionados con las condiciones externas para emprender como el caso del capital y la existencia de oportunidades para que se pueda concretar el emprendimiento, pero no hay un enfoque suficiente desde la lógica de los procesos cognitivos y las capacidades de las personas (Welter, Baker \& Wirsching, 2017). La capacidad emprendedora es la habilidad que tiene el individuo para tomar decisiones con iniciativa propia y mantenerlas las mismas con perseverancia mientras su entorno cambia (Majmud, 2013). Además, existen 3 pilares que fundamentan la capacidad de emprender: tener ideas propias, utilizar estas ideas para identificar oportunidades y saber organizar los recursos para ejecutar la idea inicial. Para Yépez, Frías \& Asunción (2019) los emprendedores tienen la capacidad hacer que las ideas iniciales en modelos de negocio rentables que aporten positivamente a la sociedad. Los tipos de capacidades para emprender que tiene un individuo pueden ser: proactividad, toma de decisiones, inteligencia emocional, valores y planificación (Roegiers, 2000). Para Schmutzler, Andonova y Diaz-Serrano (2019), poseer capacidades para el emprendimiento, y específicamente aquellas que se relacionan con la autoeficacia, son claves para el éxito de iniciar un emprendimiento. De esta forma, las capacidades resultan claves para poder establecer premisas claves para el emprendimiento, las que, unidas a las condiciones para emprender, constituyen la tercera ola de estudios del emprendimiento (Welter et al., 2019).

\section{Género y emprendimiento}

Autores como Kuratko y Morris (2018) argumentan el emprendimiento se encuentra permeado por variables sociodemográficas, siendo el género una de ellas. Según $\operatorname{INCAE}^{1}$, la discriminación de género prevalece en las sociedades de América Latina, donde, las mujeres se ven afectadas directamente en la participación en el emprendimiento. Además, limita su crecimiento personal y su capacidad económica para el desarrollo de su entorno familiar (Ilie \& Cardoza, 2018). Mediante el estudiado elaborado por Henríquez, Mosquera \& Arias (2010), determinan que existe una diferencia entre hombres y mujeres emprendedoras, también se determina que las mujeres son más emprendedoras que los hombres, sin embargo, las mujeres presentan diferencias significativas en los ingresos y posibilidades que pueden acceder frente a los hombres. También se determina que la brecha emprendedora entre hombres y mujeres en los recientes años cada vez se acorta más (Pinokvetskaia \& Ginzburg, 2018).

\section{Contexto académico y emprendimiento}

Según Kuratko (2005) consideraba que el emprendimiento o ciertas dimensiones de este no pueden enseñarse. Por otro lado, mediante el estudio realizado por Powell (2013) se determina que la premisa es obsoleta ya que hoy en día se puede enseñar habilidades, capacidades y conocimiento sobre emprender, sobre todo en el campo universitario, ya que el modelo triple hélice (universidad-industria-gobierno) promueve que los estudiantes puedan ser capaces de aportar a la sociedad con su conocimiento, esto puede evidenciar principalmente con una idea emprendedora. Mediante un estudio realizado por Astudillo, Mora \& Pozo (2019) se determina que los estudiantes tienen un aumento en sus habilidades, conocimientos e información al finalizar un periodo académico en comparación al inicio de este. Esto les

\footnotetext{
${ }^{1}$ Instituto Centroamericano de Administración de Empresas
} 
permite a los estudiantes acceder a mayor información y aumentar la posibilidad de emprender. Sin embargo, a pesar de que existen estudios previos de la relación entre los ambientes de innovación y el emprendimiento, todavía hay espacios para poder explicar la influencia de la cultura y los contextos en el emprendimiento (Lounsbury, Cornelissen, Granqvist \& Grodal, 2019).

Por esta razón, el objetivo de la presente investigación es identificar la relación entre las capacidades e interés de emprender de jóvenes estudiantes de la carrera de ingeniería empresarial y jóvenes miembros de la AIESEC del Ecuador con respecto a su género. De esta manera se estarían considerando aspectos relevantes para comprender el emprendimiento como el género y el contexto.

\section{Muestra}

\section{Metodología}

El estudio se realizó en dos organizaciones relacionadas con estudiantes universitarios. Una de las organizaciones fue la Facultad de Gestión de una Universidad Pública ( $N=136)$ y en el otro caso fue en una asociación de estudiantes internacional $(\mathrm{N}=106)$. En ambas organizaciones se consideraron estudiantes ecuatorianos, en su mayoría solteros, con un promedio de edad de 21 años.

\section{Herramientas}

Las herramientas para hacer las mediciones se basaron en el estudio previo de Ramos, Ruiz \& Franco-crespo, 2020), permitiendo la evaluación de intereses de emprendimiento, divididos en capacidad de emprender, percepciones sobre la creación de empresas e influencias para emprender. Asimismo, en las competencias relacionadas con el emprendimiento se consideraron los valores, la esperanza, la autoestima, la personalidad proactiva, la actitud hacia el emprendimiento, la toma de decisiones y la empatía.

\section{Procedimiento}

Para el estudio se aplicó una encuesta utilizando la plataforma Google Forms a los dos grupos de estudiantes. La encuesta estuvo online durante quince días, con seguimiento por parte de los investigadores como forma de recordatorio para el llenado. El instrumento fue anónimo y la participación de los estudiantes fue de forma voluntaria.

\section{Análisis de los datos}

Los datos fueron analizados utilizando cálculos de frecuencia y comparaciones de media a partir del coeficiente ANOVA. Igualmente se realizaron correlaciones entre variables utilizando para ellos el coeficiente de Spearman.

\section{Resultados}

Los valores se obtuvieron considerando comparaciones generales entre los dos grupos de estudiantes, así como comparaciones teniendo en cuenta el género del estudiante. A continuación, se presentan los resultados obtenidos.

\section{Diferencias entre grupos de estudiantes teniendo en cuenta la organización}

Teniendo en cuenta a la organización de los estudiantes, se realizaron cálculos de comparación. Estos resultados se muestran en la Tabla 1. 
Tabla 1

Diferencias entre estudiantes relacionadas con las condiciones para emprender de acuerdo con su organización

\begin{tabular}{|c|c|c|c|c|c|}
\hline & Media IE & Media AIESEC & Sig. & Mínimo & Máximo \\
\hline \multicolumn{6}{|l|}{ Capacidad de emprender } \\
\hline Relación con los stakeholders & 3,664 & 4,13 & 0,000 & 2,00 & 5,00 \\
\hline Cultura de trabajo & 3,720 & 3,95 & 0,008 & 2,00 & 5,00 \\
\hline Trabajo con incertidumbre & 3,552 & 4,13 & 0,000 & 1,67 & 5,00 \\
\hline \multicolumn{6}{|l|}{ Percepciones sobre la creación de empresas } \\
\hline $\begin{array}{l}\text { En el caso que identificara posibilidades de } \\
\text { comercializar los resultados de investigación o una } \\
\text { idea consideraría seriamente establecer mi propia } \\
\text { empresa }\end{array}$ & 5,634 & 5,85 & 0,181 & 1,71 & 7,00 \\
\hline Apoyo del círculo cercano para crear empresa & 5,300 & 5,57 & 0,036 & 2,00 & 7,00 \\
\hline Intención de crear empresa & 5,655 & 5,87 & 0,150 & 2,00 & 7,00 \\
\hline Excelencia en el proceso de crear empresa & 5,117 & 5,46 & 0,039 & 1,00 & 7,00 \\
\hline Tolerancia al fracaso & 5,630 & 4,77 & 0,000 & 2,38 & 7,00 \\
\hline \multicolumn{6}{|l|}{ Influencias para emprender } \\
\hline $\begin{array}{l}\text { Mis contactos o discusiones con potenciales clientes } \\
\text { me pueden aportar información que me ayuda o } \\
\text { incentiva a crear una nueva empresa. }\end{array}$ & 4,525 & 5,88 & 0,000 & 1,00 & 7,00 \\
\hline Información y relaciones influyen a crear una empresa & 4,814 & 5,95 & 0,000 & 1,00 & 7,00 \\
\hline Mi comunidad influye para crear una empresa & 5,395 & 5,25 & 0,366 & 1,83 & 7,00 \\
\hline
\end{tabular}

Los resultados muestran que los estudiantes de AIESEC reconocen tener mejores condiciones relacionadas con el emprendimiento, así como un mejor sistema de soporte y apoyo para la creación de empresas. Sin embargo, los valores de ambos grupos de estudiantes no son elevados, lo que demuestra que hay oportunidades de mejora para que el sistema en el que se encuentran los estudiantes pueda tener un mayor impacto para el emprendimiento. Los estudiantes de administración, en cambio, mostraron valores más altos de manera estadísticamente significativa en los relacionado con la tolerancia al fracaso, lo cuál puede ser un reflejo de la cultura organizacional de la Universidad a la que pertenecen. Estos resultados de comparación, teniendo en cuenta las competencias de innovación, se muestran en la Tabla 2 .

Con relación a las competencias asociadas al emprendimiento, hubo resultados de medias más altos y estadísticamente significativos en el caso de los estudiantes de administración para las variables esperanza y autoestima. Por su parte, los estudiantes de AIESEC tuvieron valores más elevados y estadísticamente significativos comparados con los estudiantes de administración en las variables asociadas a la empatía, la actitud al emprendimiento y la proactividad. Hubo aspectos relacionados con atención y reconocimiento de los sentimientos que aparecieron de manera indistinta tanto en estudiantes de administración como en estudiantes miembros de AIESEC. 


\section{Tabla 2}

Diferencias entre estudiantes relacionadas con las competencias para emprender de acuerdo con su organización

\begin{tabular}{|c|c|c|c|c|c|}
\hline & Media IE & Media AIESEC & Sig. & Mínimo & Máximo \\
\hline \multicolumn{6}{|l|}{ Valores } \\
\hline $\begin{array}{l}\text { Pertenecer a un grupo y ser participe en ayudar a los } \\
\text { demás }\end{array}$ & 5,853 & 6,07 & 0,079 & 2,00 & 7,00 \\
\hline \multicolumn{6}{|l|}{ Esperanza } \\
\hline Esperanza & 4,689 & 4,35 & 0,026 & 1,00 & 7,00 \\
\hline \multicolumn{6}{|l|}{ Autoestima } \\
\hline Alta autoestima & 5,586 & 3,58 & 0,000 & 1,33 & 7,00 \\
\hline Baja autoestima & 5,611 & 2,16 & 0,000 & 2,00 & 7,00 \\
\hline \multicolumn{6}{|l|}{ Personalidad Proactiva } \\
\hline Personalidad Proactiva & 4,040 & 5,75 & 0,000 & 2,00 & 5,00 \\
\hline \multicolumn{6}{|l|}{ Inteligencia Emocional } \\
\hline Presto mucha atención a los sentimientos. & 2,135 & 4,22 & 0,000 & 1,00 & 4,00 \\
\hline Reconocimiento de sentimientos y emociones propios & 3,383 & 3,97 & 0,000 & 1,00 & 4,00 \\
\hline Atención a sentimientos y emociones & 5,846 & 4,24 & 0,000 & 3,00 & 7,00 \\
\hline Grado de positivismo & 3,718 & 4,12 & 0,001 & 1,00 & 5,00 \\
\hline Percepción e influencia de estado de ánimo & 3,836 & 3,67 & 0,206 & 1,33 & 5,00 \\
\hline Estado de ánimo & 3,477 & 3,66 & 0,136 & 1,00 & 5,00 \\
\hline \multicolumn{6}{|l|}{ Actitud de emprendimiento } \\
\hline Detección y aprovechamiento de oportunidades & 3,964 & 4,37 & 0,000 & 1,75 & 5,00 \\
\hline Actitud ante oportunidades y adversidad & 3,280 & 4,54 & 0,000 & 1,57 & 4,00 \\
\hline Excelencia profesional & 3,210 & 4,54 & 0,000 & 1,25 & 4,00 \\
\hline \multicolumn{6}{|l|}{ Toma de decisiones } \\
\hline Toma de decisiones & 3,235 & 6,14 & 0,000 & 1,00 & 7,00 \\
\hline \multicolumn{6}{|l|}{ Empatía } \\
\hline $\begin{array}{l}\text { Suelo darme cuenta rápidamente de cuándo un amigo/a } \\
\text { está enfadado. }\end{array}$ & 5,739 & 4,51 & 0,000 & 2,50 & 7,00 \\
\hline Detección de estado de ánimo de los demás & 3,768 & 4,31 & 0,000 & 1,00 & 5,00 \\
\hline Empatía & 3,204 & 3,72 & 0,000 & 1,00 & 5,00 \\
\hline
\end{tabular}

Nota: IE son las siglas de Ingeniería Empresarial

\section{Diferencias de género teniendo en cuenta las competencias y condiciones para el emprendimiento}

Las mismas comparaciones se realizaron teniendo en cuenta el género de los estudiantes. Las respuestas se encuentran en la Tabla 3. 


\section{Tabla 3}

Diferencias entre estudiantes relacionadas con las competencias y condiciones para emprender de acuerdo con el género

\begin{tabular}{|c|c|c|c|c|c|c|}
\hline & \multicolumn{3}{|c|}{ AIESEC } & \multicolumn{3}{|c|}{ IE } \\
\hline & Femenino & Masculino & Sig. & Femenino & Masculino & Sig. \\
\hline $\begin{array}{l}\text { Excelencia en el proceso de crear } \\
\text { empresa }\end{array}$ & 5,1564 & 5,5065 & 0,056 & 5,64 & 5,52 & 0,535 \\
\hline $\begin{array}{l}\text { Apoyo del círculo cercano para crear } \\
\text { empresa }\end{array}$ & 5,6635 & 5,6420 & 0,916 & 6,20 & 5,62 & 0,012 \\
\hline $\begin{array}{l}\text { Negocios previos y relaciones con } \\
\text { inversores influyen a crear empresa }\end{array}$ & 4,5705 & 5,1667 & 0,019 & 6,03 & 5,88 & 0,512 \\
\hline Lealtad y honestidad & 5,9244 & 5,7500 & 0,334 & 5,89 & 6,21 & 0,045 \\
\hline Baja autoestima & 2,1032 & 2,1811 & 0,607 & 3,96 & 4,42 & 0,017 \\
\hline Proactividad & 5,7560 & 5,9776 & 0,139 & 3,99 & 4,43 & 0,004 \\
\hline $\begin{array}{l}\text { Detección y aprovechamiento de } \\
\text { oportunidades }\end{array}$ & 3,1513 & 3,2943 & 0,089 & 4,39 & 4,65 & 0,020 \\
\hline Empatía & 3,2733 & 3,1053 & 0,347 & 3,48 & 3,91 & 0,035 \\
\hline
\end{tabular}
género. Las variables y valores que no tuvieron diferencias estadísticamente significativas no están incluidos en la Tabla 3 . IE son las siglas de Ingeniería Empresarial.

El comportamiento relacionado con diferencias de género fue distinto en el caso de estudiantes miembros de AIESEC en comparación con los estudiantes de administración. En el caso de AIESEC, hubo diferencias estadísticamente significativas relacionadas con el género teniendo en cuenta la percepción de excelencia al momento de crear una empresa y la existencia de negocios previos, donde el género masculino tuvo puntuaciones más altas. Esta diferencia de comportamientos, donde el género masculino tuvo una puntuación más alta, también se reflejó en el caso de los estudiantes de administración. Las personas de género masculino indicaron tener mayores niveles de lealtad, honestidad, proactividad, empatía y aprovechamiento de oportunidades. Las personas de género femenino, por su parte, mostraron tener una autoestima más baja, pero mayor apoyo del círculo cercano al momento de crear una empresa.

\section{Relaciones entre competencias y condiciones para el emprendimiento de acuerdo con el género}

Para poder comprender la relación entre las competencias y las condiciones para el emprendimiento de acuerdo con el género, se realizaron cálculos para cada una de las organizaciones a las que pertenecen los estudiantes. Los resultados para el género femenino en el caso de estudiantes de administración se encuentran en la Tabla 4.

En el caso del género femenino en los estudiantes de administración, la autoestima a pesar de ser uno de los atributos claves para el emprendimiento, fue la variable con el menor número de correlaciones estadísticamente significativas con los elementos relacionados con las condiciones para emprender. Los otros elementos con menor número de correlaciones fueron el ser admirado y la empatía. 


\section{Tabla 4}

Relación entre competencias y condiciones para emprender de acuerdo en estudiantes de administración de acuerdo con el género

\begin{tabular}{|c|c|c|c|c|c|c|c|c|c|c|}
\hline & \multicolumn{10}{|c|}{ Condiciones de emprendimiento } \\
\hline & 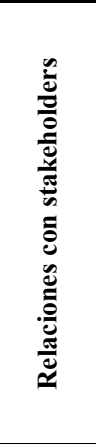 & 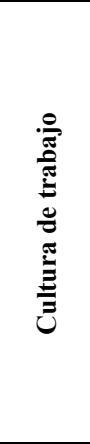 & 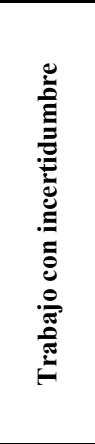 & 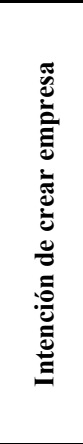 & 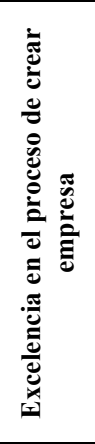 & 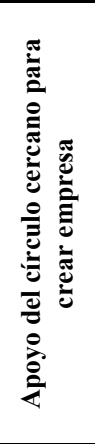 & 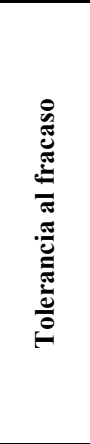 & 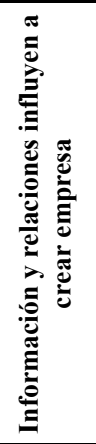 & 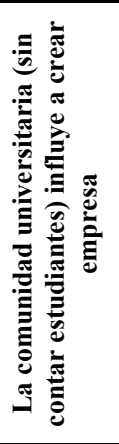 & 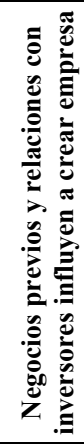 \\
\hline \multicolumn{11}{|l|}{ Género Femenino } \\
\hline \multicolumn{11}{|l|}{$\begin{array}{l}\text { Competencias de } \\
\text { emprendimiento }\end{array}$} \\
\hline $\begin{array}{l}\text { Pertenecer a un grupo y } \\
\text { ser partícipe en ayudar a } \\
\text { los demás }\end{array}$ & ,222 & ,209 & $248^{*}$ & $414^{* *}$ & $318^{* *}$ & $298^{* *}$ & $280^{*}$ & $512^{* *}$ & $434^{* *}$ &, $324^{* *}$ \\
\hline Lealtad y honestidad & $319^{* *}$ & $318^{* *}$ & $279^{*}$ & $262^{*}$ & $301^{* *}$ & $405^{* *}$ & $309^{* *}$ & $405^{* *}$ &, 151 & 0,114 \\
\hline Ser admirado &, 054 &, 188 &, 085 &, 127 &, 069 &, 117 & $392^{* *}$ &, 135 & $357^{* *}$ &, $241^{*}$ \\
\hline Autenticidad & $316^{* *}$ & $424^{* *}$ & $276^{*}$ & $298^{* *}$ & $511^{* *}$ & $263^{*}$ & $462^{* *}$ & $486^{* *}$ & $331^{* *}$ &, $366^{* *}$ \\
\hline $\begin{array}{l}\text { Comportamiento referente } \\
\text { al contexto }\end{array}$ & $276^{*}$ & $411^{* *}$ &, 178 & $279^{*}$ & $419^{* *}$ & $351^{* *}$ & $490^{* *}$ & $410^{* *}$ & $264^{*}$ &, $280^{*}$ \\
\hline Esperanza & $327^{* *}$ & $384^{* *}$ & $259^{*}$ & $373^{* *}$ & $482^{* *}$ & $263^{*}$ & $320^{* *}$ & $421^{* *}$ & $342^{* *}$ &, $263^{*}$ \\
\hline Baja autoestima & 0,174 & 0,102 &, $260^{*}$ &, $224^{*}$ & 0,126 & 0,128 &, 116 & 0,162 & $270^{*}$ & 0,070 \\
\hline Alta autoestima &, 106 & ,194 &, 108 &, 172 & $284^{*}$ &, 129 &, 125 & $381^{* *}$ &, 061 & 0,183 \\
\hline Proactividad & $328^{* *}$ & $270^{*}$ & $237^{*}$ & $289^{*}$ & $516^{* *}$ & $416^{* *}$ & $356^{* *}$ & $483^{* *}$ & $338^{* *}$ &, $408^{* *}$ \\
\hline $\begin{array}{l}\text { Reconocimiento de } \\
\text { sentimientos y emociones } \\
\text { propios }\end{array}$ & $443^{* *}$ & $386^{* *}$ & $373^{* *}$ & $268^{*}$ & $393^{* *}$ & $259^{*}$ & $271^{*}$ & $391^{* *}$ & $252^{*}$ & 0,200 \\
\hline Grado de positivismo & $392^{* *}$ & $258^{*}$ & $425^{* *}$ & $238^{*}$ & $247^{*}$ &, 204 &, 185 & $393^{* *}$ & $306^{* *}$ & 0,205 \\
\hline $\begin{array}{l}\text { Percepción e influencia de } \\
\text { estado de ánimo }\end{array}$ & $280^{*}$ & $321^{* *}$ &, 210 &, 118 & $228^{*}$ &, 122 & $322^{* *}$ & $363^{* *}$ & $333^{* *}$ &, $261^{*}$ \\
\hline $\begin{array}{l}\text { Atención a sentimientos y } \\
\text { emociones }\end{array}$ & $505^{* *}$ & $340^{* *}$ & $303^{* *}$ & $355^{* *}$ & $283^{*}$ & $374^{* *}$ &, 217 & $338^{* *}$ & ,206 & 0,161 \\
\hline $\begin{array}{l}\text { Actitud ante } \\
\text { oportunidades y } \\
\text { adversidad }\end{array}$ & $252^{*}$ & $252^{*}$ & $283^{*}$ & ,209 & $285^{*}$ &, 218 &, 101 & $431^{* *}$ & $227^{*}$ &, $305^{* *}$ \\
\hline $\begin{array}{l}\text { Detección y } \\
\text { aprovechamiento de } \\
\text { oportunidades }\end{array}$ & $374^{* *}$ &, 181 & $254^{*}$ & $246^{*}$ & $336^{* *}$ & $266^{*}$ & $253^{*}$ & $390^{* *}$ &, 220 &, $262^{*}$ \\
\hline Excelencia profesional & $408^{* *}$ & $257^{*}$ & $380^{* *}$ & $364^{* *}$ & $359^{* *}$ & $447^{* *}$ & ,139 & $487^{* *}$ & ,137 &, $328^{* *}$ \\
\hline $\begin{array}{l}\text { Capacidad de planificar y } \\
\text { tomar decisiones }\end{array}$ & $234^{*}$ & $337^{* *}$ & $293^{*}$ & $284^{*}$ & $427^{* *}$ & $340^{* *}$ & $370^{* *}$ & $535^{* *}$ & $362^{* *}$ &, $414^{* *}$ \\
\hline
\end{tabular}




\begin{tabular}{|c|c|c|c|c|c|c|c|c|c|c|}
\hline $\begin{array}{l}\text { Detección de estado de } \\
\text { ánimo de los demás }\end{array}$ & $233^{*}$ & $364^{* *}$ & $268^{*}$ & , 190 & $250^{*}$ & $273^{*}$ & $249^{*}$ & $410^{* *}$ & ,222 & 0,173 \\
\hline Empatía &, 016 & 187 & 0,041 &, 119 & $240^{*}$ & ,190 & $235^{*}$ & $333^{* *}$ & $450^{* *}$ &, $316^{* *}$ \\
\hline \multicolumn{11}{|l|}{ Género Masculino } \\
\hline \multicolumn{11}{|l|}{$\begin{array}{l}\text { Competencias de } \\
\text { emprendimiento }\end{array}$} \\
\hline $\begin{array}{l}\text { Pertenecer a un grupo y } \\
\text { ser partícipe en ayudar a } \\
\text { los demás }\end{array}$ & ,196 & $295^{*}$ &, 220 & $421 * *$ & $316^{*}$ & $480 * *$ & $394 * *$ & $554 * *$ & $477 * *$ & 0,175 \\
\hline Lealtad y honestidad & ,094 &, 151 & ,165 & ,231 &, 139 & $412 * *$ & $347^{*}$ & $475^{* *}$ & ,253 & 0,232 \\
\hline Ser admirado &, 171 & $367 * *$ & $275^{*}$ &, 081 & $340^{*}$ & ,202 & ,255 &, 179 & $466^{* *}$ & 0,151 \\
\hline Autenticidad & $381 * *$ & $349 * *$ & $284 *$ & $385 * *$ & $417^{* *}$ &, 235 & ,192 & $351 * *$ &, 020 & 0,241 \\
\hline $\begin{array}{l}\text { Comportamiento referente } \\
\text { al contexto }\end{array}$ & 215 & $350 * *$ & ,160 & $507^{* *}$ & $349 * *$ & $382 * *$ & $372 * *$ & $492 * *$ & $383^{* *}$ & 0,081 \\
\hline Esperanza & $446^{* *}$ & $377 * *$ & $471 * *$ & $445^{* *}$ & $473 * *$ & $438 * *$ & $360 * *$ & $539 * *$ &, 258 &, $400 * *$ \\
\hline Baja autoestima & 0,185 & ,156 & 0,222 & 0,186 & 0,163 &, 031 & 0,060 & 0,196 & 100 & $-0,141$ \\
\hline Alta autoestima & $403 * *$ &, 138 & $349^{*}$ & $380 * *$ & $354 * *$ & $371 * *$ &, 156 & $374 * *$ &, 256 & 0,262 \\
\hline Proactividad & $411^{* *}$ & ,239 & $361 * *$ & $471^{* *}$ & $478 * *$ & $397 * *$ & $284^{*}$ & $409 * *$ & 165 &, $352 * *$ \\
\hline $\begin{array}{l}\text { Reconocimiento de } \\
\text { sentimientos y emociones } \\
\text { propios }\end{array}$ &, 174 &, 145 & $293^{*}$ & $374 * *$ & ,243 & $374 * *$ & ,200 &, 217 & $327^{*}$ & 0,192 \\
\hline Grado de positivismo & $275^{*}$ & ,260 & ,266 & $362 * *$ & $277^{*}$ & $356^{* *}$ & $358 * *$ & $446^{* *}$ & $278^{*}$ & 0,226 \\
\hline $\begin{array}{l}\text { Percepción e influencia de } \\
\text { estado de ánimo }\end{array}$ &, 119 &, 080 &, 032 & ,198 &, 212 &, 100 &, 104 &, 098 & ,272 & 0,107 \\
\hline $\begin{array}{l}\text { Atención a sentimientos y } \\
\text { emociones }\end{array}$ & $297^{*}$ & ,136 & ,205 & $442 * *$ & ,264 & ,203 &, 130 & $354 *$ &, 175 & 0,160 \\
\hline $\begin{array}{l}\text { Actitud ante } \\
\text { oportunidades y } \\
\text { adversidad }\end{array}$ & ,225 &, 050 & $290^{*}$ &, 105 & $296^{*}$ &, 148 & 0,075 &, 125 & 0,097 & 0,257 \\
\hline $\begin{array}{l}\text { Detección y } \\
\text { aprovechamiento de } \\
\text { oportunidades }\end{array}$ & $478 * *$ & $372 * *$ & $472 * *$ & $319^{*}$ & ,267 & ,244 &, 035 & $287 *$ &, 044 &, $340^{*}$ \\
\hline Excelencia profesional &, 148 & 0,007 &, 173 &, 066 & ,207 & ,241 & ,078 &, 179 &, 030 & 0,126 \\
\hline $\begin{array}{l}\text { Capacidad de planificar y } \\
\text { tomar decisiones }\end{array}$ & ,198 & ,217 & $289^{*}$ & $347 *$ & $285^{*}$ & ,265 & ,204 & $412 * *$ & $337^{*}$ &, $345^{*}$ \\
\hline $\begin{array}{l}\text { Detección de estado de } \\
\text { ánimo de los demás }\end{array}$ & $307^{*}$ & $301 *$ & $354 * *$ & 220 & ,244 & ,222 & ,041 & ,192 & $275^{*}$ & 0,180 \\
\hline Empatía & ,146 & ,141 & ,112 & 0,019 &, 111 & ,158 & $324 *$ & ,092 & $596^{* *}$ & 0,109 \\
\hline
\end{tabular}

El género masculino en estudiantes de administración tuvo menos componentes de comportamientos que correlacionaron de forma estadísticamente significativa con las condiciones para emprender. Los que no tuvieron correlaciones significativas fueron la percepción de la influencia y la baja autoestima. Por su parte, la honestidad, el estado de ánimo, la excelencia profesional, la atención a sentimientos, la capacidad de detectar estados de ánimo 
en las personas y la empatía tuvieron pocas correlaciones estadísticamente significativas. En el caso de las estudiantes de género femenino de AIESEC, los cálculos de correlaciones están presentados en la Tabla 5.

\section{Tabla 5}

Relación entre competencias y condiciones para emprender de acuerdo en estudiantes de AIESEC de acuerdo con el género

\begin{tabular}{|c|c|c|c|c|c|c|c|c|c|}
\hline 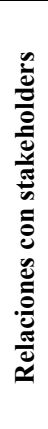 & 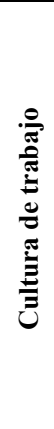 & 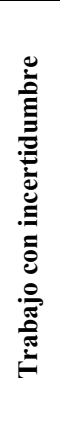 & 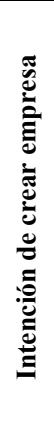 & 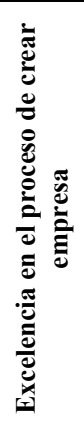 & 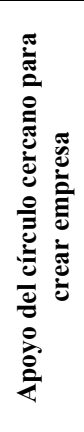 & 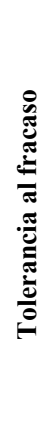 & 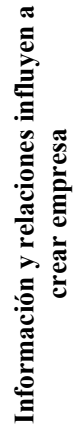 & 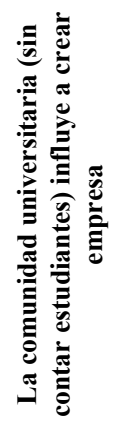 & 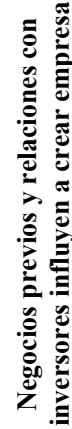 \\
\hline
\end{tabular}

\section{Género Femenino}

\begin{tabular}{|c|c|c|c|c|c|c|c|c|c|c|}
\hline $\begin{array}{l}\text { Competencias de } \\
\text { emprendimiento }\end{array}$ & & & & & & & & & & \\
\hline $\begin{array}{l}\text { Pertenecer a un grupo y ser } \\
\text { partícipe en ayudar a los } \\
\text { demás }\end{array}$ &, 233 &, 041 &, 126 & ,201 &, 169 & ,201 &, 104 & ,211 &, 134 & 0,223 \\
\hline Lealtad y honestidad & $344 * *$ & $351^{* *}$ & ,206 & $361^{* *}$ & $340 * *$ & ,221 & $317^{*}$ & $309^{*}$ & $274 *$ & 0,194 \\
\hline Ser admirado & ,159 & 0,034 & ,158 &, 036 &, 047 & 0,023 &, 025 &, 065 & 0,102 & $-0,041$ \\
\hline Autenticidad &, $272 *$ & 0,002 & 0,209 & 0,089 & 0,089 & 0,123 & 0,165 & 0,052 & 0,015 & $-0,146$ \\
\hline $\begin{array}{l}\text { Comportamiento referente al } \\
\text { contexto }\end{array}$ & ,075 & $296^{*}$ & 0,116 & ,226 & ,193 &, 117 & $333^{*}$ & $362 * *$ &, 134 & 0,133 \\
\hline Esperanza & ,207 &, 111 &, 056 & $275^{*}$ & $365 * *$ & $308^{*}$ & ,175 & $292 *$ & ,228 &, $339 * *$ \\
\hline Baja autoestima &, 143 &, 065 &, 129 & ,244 & $291 *$ & $292 *$ & $438 * *$ & ,215 & ,066 & 0,005 \\
\hline Alta autoestima &, 052 & 0,137 &, 031 & $305^{*}$ & $320^{*}$ & $278^{*}$ & $272 *$ & $299^{*}$ &, 068 & 0,184 \\
\hline Proactividad &, 251 &, 128 & ,239 & $356^{* *}$ & $495 * *$ & $613 * *$ & $439 * *$ & $457 * *$ & $375^{* *}$ &, $359 * *$ \\
\hline $\begin{array}{l}\text { Reconocimiento de } \\
\text { sentimientos y emociones } \\
\text { propios }\end{array}$ &, 056 & 0,073 & 0,102 &, 118 & 0,024 & 0,011 &, 117 &, 159 & ,094 & 0,019 \\
\hline Grado de positivismo &, 065 &, 067 &, 035 & ,217 &, 170 & ,203 & ,204 & $364 * *$ &, 092 & 0,194 \\
\hline $\begin{array}{l}\text { Percepción e influencia de } \\
\text { estado de ánimo }\end{array}$ & ,236 & ,248 & 088 & $379 * *$ & $571 * *$ & $521 * *$ & $602 * *$ & $480 * *$ &, 167 &, $275^{*}$ \\
\hline $\begin{array}{l}\text { Atención a sentimientos y } \\
\text { emociones }\end{array}$ & $334 * *$ & $382 * *$ & $281^{*}$ & $338 * *$ & $519 * *$ & $399 * *$ & $512 * *$ & $308^{*}$ & ,239 & ,299* \\
\hline $\begin{array}{l}\text { Actitud ante oportunidades y } \\
\text { adversidad }\end{array}$ & ,195 & ,219 &, 218 &, 154 & $422 * *$ & $300^{*}$ & $335 * *$ & $279^{*}$ & ,198 & 0,238 \\
\hline $\begin{array}{l}\text { Detección y } \\
\text { aprovechamiento de } \\
\text { oportunidades }\end{array}$ & $335 * *$ &, 235 &, 057 &, 183 & $334 * *$ &, 254 & $346^{* *}$ & $269^{*}$ &, 018 & 0,093 \\
\hline Excelencia profesional & $364 * *$ & ,105 & ,253 & ,006 & ,099 & ,087 & ,009 & 0,018 & 0,016 & 0,027 \\
\hline
\end{tabular}




\begin{tabular}{|c|c|c|c|c|c|c|c|c|c|c|}
\hline $\begin{array}{l}\text { Capacidad de planificar y } \\
\text { tomar decisiones }\end{array}$ & ,249 &, 148 & $280^{*}$ &, 059 &, 220 &, 163 & ,209 & ,009 &, 177 & 0,220 \\
\hline $\begin{array}{l}\text { Detección de estado de } \\
\text { ánimo de los demás }\end{array}$ &, 139 &, 048 &, 032 &, 125 &, 078 &, 197 & ,066 &, 144 & 0,119 & 0,078 \\
\hline Empatía &, 233 &, 041 & ,126 & ,201 &, 169 & ,201 & ,104 & ,211 & ,134 & 0,223 \\
\hline \multicolumn{11}{|l|}{ Género Masculino } \\
\hline \multicolumn{11}{|l|}{$\begin{array}{l}\text { Competencias de } \\
\text { emprendimiento }\end{array}$} \\
\hline $\begin{array}{l}\text { Pertenecer a un grupo y ser } \\
\text { partícipe en ayudar a los } \\
\text { demás }\end{array}$ &, 230 & $300^{*}$ & $352 *$ & $420 * *$ & $446^{* *}$ &, 137 & ,188 &, 018 &, 002 & 0,230 \\
\hline Lealtad y honestidad & ,286 & $306^{*}$ & $342^{*}$ & ,008 &, 014 & 0,020 & ,265 &, 027 & $401 * *$ &, $314 *$ \\
\hline Ser admirado &, 019 &, 133 &, 194 & 0,037 &, 129 &, 012 & ,218 & 0,185 &, 110 & 0,093 \\
\hline Autenticidad & 0,057 & 0,028 & 0,221 & 0,100 & 0,080 & 0,039 & 0,193 &, 109 &, $295^{*}$ & $-0,110$ \\
\hline $\begin{array}{l}\text { Comportamiento referente al } \\
\text { contexto }\end{array}$ &, 085 &, 235 &, 232 &, 185 & $315^{*}$ &, 037 & $354^{*}$ &, 147 &, 053 & $-0,007$ \\
\hline Esperanza & $424 * *$ & $342 *$ & $506^{* *}$ & $405^{* *}$ & $413 * *$ &, 259 & $297^{*}$ &, 181 &, 197 &, $318^{*}$ \\
\hline Baja autoestima & $360^{*}$ & ,229 & $392 * *$ & ,267 &, 252 &, 134 & $351 *$ & $308^{*}$ & $365^{*}$ & 0,260 \\
\hline Alta autoestima & $505 * *$ & $447 * *$ & $563 * *$ & $396^{* *}$ & $422 * *$ &, 144 & $309 *$ &, 229 &, 198 & 0,271 \\
\hline Proactividad & $530 * *$ & $490 * *$ & $634 * *$ & $481 * *$ & $425^{* *}$ & ,219 & $354^{*}$ & $344^{*}$ & ,198 & 0,271 \\
\hline $\begin{array}{l}\text { Reconocimiento de } \\
\text { sentimientos y emociones } \\
\text { propios }\end{array}$ & $538 * *$ & $503 * *$ & $404 * *$ & $374 *$ & ,246 &, 104 &, 180 & $306^{*}$ &, 141 & 0,108 \\
\hline Grado de positivismo & $450 * *$ & $622 * *$ & $354^{*}$ & $542 * *$ & $372 *$ &, 162 & $408 * *$ &, 259 &, 190 & 0,135 \\
\hline $\begin{array}{l}\text { Percepción e influencia de } \\
\text { estado de ánimo }\end{array}$ & $305^{*}$ & $472 * *$ & $439 * *$ & $476^{* *}$ & $505^{* *}$ & $374 *$ & $351^{*}$ &, 069 &, 213 &, $412 * *$ \\
\hline $\begin{array}{l}\text { Atención a sentimientos y } \\
\text { emociones }\end{array}$ &, 246 & $333^{*}$ & $298^{*}$ & $344^{*}$ & $338^{*}$ &, 267 & $405^{* *}$ &, 180 & $365^{*}$ &, $357^{*}$ \\
\hline $\begin{array}{l}\text { Actitud ante oportunidades y } \\
\text { adversidad }\end{array}$ & $354^{*}$ & $433 * *$ & $375^{*}$ & $498 * *$ & $471 * *$ & $332 *$ & $436^{* *}$ & $294 *$ &, 274 &, $453^{* *}$ \\
\hline $\begin{array}{l}\text { Detección y } \\
\text { aprovechamiento de } \\
\text { oportunidades }\end{array}$ & $373^{*}$ & $446^{* *}$ & $394 * *$ & $542 * *$ & $487 * *$ & $330^{*}$ & $629 * *$ & $454 * *$ & $299 *$ &, $323^{*}$ \\
\hline Excelencia profesional & ,208 & ,236 &, 177 & $356^{*}$ & ,290 & $339 *$ & ,208 & ,135 & ,194 & 0,242 \\
\hline $\begin{array}{l}\text { Capacidad de planificar y } \\
\text { tomar decisiones }\end{array}$ & $362^{*}$ & $408 * *$ & $354^{*}$ & $510^{* *}$ & $393 * *$ & $448 * *$ & $351 *$ & $310^{*}$ & ,255 &, $369^{*}$ \\
\hline $\begin{array}{l}\text { Detección de estado de } \\
\text { ánimo de los demás }\end{array}$ & ,199 & ,261 & 0,072 & ,268 & ,282 &, 128 & ,116 & ,292 & 0,020 & 0,135 \\
\hline Empatía & ,230 & $300^{*}$ & $352^{*}$ & $420 * *$ & $446^{* *}$ & ,137 & ,188 &, 018 & ,002 & 0,230 \\
\hline
\end{tabular}

Las estudiantes de género femenino de AIESEC tuvieron menos aspectos relacionados con las competencias para el emprendimiento en comparación con las estudiantes de 
administración. En este caso hubo aspectos que no correlacionaron de manera significativa con ninguno de los elementos asociados a condiciones de emprendimiento como el caso de la inteligencia emocional, el hecho de pertenecer a un grupo y el ser admirado.

El comportamiento que tuvieron los estudiantes de género masculino de AIESEC, con relación a los elementos que no correlacionaron de forma estadísticamente significativa, en cambio, fue similar al de los estudiantes de administración de género masculino. Igualmente aparece la capacidad de detectar estados de ánimo como uno de los elementos que no correlacionó de forma estadísticamente significativa. Adicional, se encuentra el hecho de ser admirado como otro de los elementos que no tuvo este tipo de correlaciones. Adicional, podemos identificar que la honestidad, y el comportamiento referente al contexto fueron dos elementos que correlacionaron de forma estadísticamente significativa solamente con uno de los elementos de las condiciones de emprendimiento.

\section{Discusión}

Los resultados presentan elementos claves que permiten comprender las diferencias que se encontraron teniendo en cuenta el género y el contexto de los estudiantes. A continuación, se discuten los principales hallazgos encontrados.

\section{El contexto es importante en el análisis del emprendimiento}

Efectivamente, autores como Rich et al., (2019) argumentan que el contexto influye en el emprendimiento, una vez que países en desarrollo van a poseer más emprendedores debido al problema del desempleo que afrontan. Asimismo, Ramos et al. (2020) indican que los contextos, influenciados por la educación, pueden orientar el comportamiento de los emprendedores hacia la búsqueda de iniciativas personales, o hacia el desarrollo económico del negocio. Por su parte, Gamal et al. (2011) indica que el contexto determina las capacidades de innovación y las expectativas que las personas tienen con relación a emprender (Bakir \& Jarvis, 2017; Gaddefors \& Anderson, 2017; Gamal et al., 2011; Jones et al., 2019; Meoli et al., 2020; Nakara et al., 2019). En este sentido, nuestros resultados evidencias que el contexto define la forma en que se ve y desarrolla el emprendimiento en las personas.

\section{Existen diferencias de género con relación a las capacidades e intereses para emprender}

Autores como Rich et al. (2019), indican que los emprendedores pueden clasificarse en varios tipos, entre ellos existe una clasificación teniendo en cuenta al género de la persona. Esto se ha visualizado en las formas de abordar temas como la innovación y el emprendimiento, una vez que las mujeres han presentado comportamientos diferenciadores en comparación a los hombres (Bjornali \& Anne Støren, 2012). Sarfaraz et al. (2014) identificó que el emprendimiento en el género femenino es independiente de si existen o no sistemas que garanticen la igualdad de género, por lo que el deseo de emprender es independiente de si la mujer se siente o no discriminada en su contexto, a pesar de que existen propuestas relacionadas con la generación de políticas de disminución de la brecha de desigualdad con relación al emprendimiento, relacionadas con el acceso a financiamiento y capital, la relación trabajo familia y los prejuicios y estereotipos que viven las mujeres en el contexto laboral, entre otros (Dauvellier et al., 2016).

Teniendo en cuentas las características y las intenciones de emprender, Sullivan \& Meek (2012) determinó que existen factores individuales, como la persistencia, que es más frecuente en mujer en comparación a sus colegas masculinos. Asimismo, Mayorga et al. (2020) determinaron que el género femenino, al momento de emprender, tienen motivaciones diferentes en comparación a los hombres, estando más orientadas a temas como la familia y siendo más realistas durante todo el proceso. Por su parte, los hombres están más motivados 
por temas económicos, sobre todo dados por la condición de género del hombre como el proveedor (Mayorga et al., 2020). En el caso de la motivación, estudios como el de Ward et al. (2019) determinaron que las mujeres, en cambio, están más motivadas que los hombres al momento de emprender, mientras que los hombres se guían más por temas subjetivos relacionados con la intención de hacer su emprendimiento.

\section{Existen diferencias de género con relación a las capacidades e intereses para emprender}

Autores como Rich et al. (2019), indican que los emprendedores pueden clasificarse en varios tipos, entre ellos existe una clasificación teniendo en cuenta al género de la persona. Esto se ha visualizado en las formas de abordar temas como la innovación y el emprendimiento, una vez que las mujeres han presentado comportamientos diferenciadores en comparación a los hombres (Bjornali \& Anne Støren, 2012). Sarfaraz et al. (2014) identificó que el emprendimiento en el género femenino es independiente de si existen o no sistemas que garanticen la igualdad de género, por lo que el deseo de emprender es independiente de si la mujer se siente o no discriminada en su contexto, a pesar de que existen propuestas relacionadas con la generación de políticas de disminución de la brecha de desigualdad con relación al emprendimiento, relacionadas con el acceso a financiamiento y capital, la relación trabajo familia y los prejuicios y estereotipos que viven las mujeres en el contexto laboral, entre otros (Dauvellier et al., 2016).

Teniendo en cuentas las características y las intenciones de emprender, Sullivan \& Meek (2012) determinó que existen factores individuales, como la persistencia, que es más frecuente en mujer en comparación a sus colegas masculinos. Asimismo, Mayorga et al. (2020) determinaron que el género femenino, al momento de emprender, tienen motivaciones diferentes en comparación a los hombres, estando más orientadas a temas como la familia y siendo más realistas durante todo el proceso. Por su parte, los hombres están más motivados por temas económicos, sobre todo dados por la condición de género del hombre como el proveedor (Mayorga et al., 2020). En el caso de la motivación, estudios como el de Ward et al. (2019) determinaron que las mujeres, en cambio, están más motivadas que los hombres al momento de emprender, mientras que los hombres se guían más por temas subjetivos relacionados con la intención de hacer su emprendimiento.

\section{Hay componentes de competencias que tienen más correlaciones que otros con relación a las condiciones para emprender, y esto es diferente de acuerdo con el género}

Por último, uno de los hallazgos encontrados está relacionados con la existencia de diferencias con relación a las competencias que se relacionan con las condiciones para emprender, las que varían de acuerdo al género y al contexto. Estos resultados se contraponen a las premisas indicadas en el estudio de Marin-Garcia et al. (2011), que justifica el hecho de que hay competencias que son universales y que, por tanto, no se diferencian atendiendo a los contextos. Sanz-Valle et al. (2011), en relación con el contexto, menciona que el emprendimiento y la innovación son característicos de un tipo de cultura adhocrática, manteniendo la lógica de que contextos semejantes van a tener comportamientos y competencias semejantes a desarrollar. Esta percepción de competencias comunes, han permitido la elaboración de modelos de competencias orientados al emprendimiento y la innovación (Ward et al., 2019). Sin embargo, nuestros resultados muestran que, si bien hay competencias que van a ser comunes en su relación con las condiciones para el emprendimiento, van a haber diferencias dadas por el género que hacen que existan unas competencias que no mantengan el comportamiento esperado, por no tener correlaciones o tener correlaciones bajas. Tal es el caso de la búsqueda de la admiración o temas como la detección de emociones en las personas. 


\section{Conclusiones}

De acuerdo con los resultados obtenidos, podemos concluir que, efectivamente hay diferencias teniendo en cuenta al género de los estudiantes, siendo los estudiantes de administración los que mayor cantidad de diferencias de género poseen en cuanto a la valoración obtenida en las variables medidas. Las mayores diferencias fueron relacionadas a la autoestima, la lealtad, la honestidad, la proactividad, la empatía y el aprovechamiento de oportunidades; elementos que fueron estadísticamente superiores en el caso de los hombres. Otras diferencias identificadas estuvieron en la relación existente entre las competencias y las condiciones para emprender. En este caso, las estudiantes de AIESEC tuvieron menos correlaciones significativas en comparación a las estudiantes de administración. Por otro lado, las estudiantes, en sentido general, tuvieron mayor número de correlaciones estadísticamente significativas en comparación a los estudiantes de género masculino.

Otro hallazgo importante fue relacionado al contexto, pues en función del espacio de relación entre los estudiantes, encontramos diferencias estadísticamente significativas. Los estudiantes de administración puntuaron más alto en aspectos como la esperanza y la autoestima, mientras que los estudiantes de AIESEC tuvieron mejores valores en temas como la empatía, la actitud al emprendimiento y la proactividad. Sin embargo, en ambas instituciones los valores de medias obtenidos fueron bajos, lo que indica oportunidades de mejora y de desarrollo de competencias, así como de creación de mejores condiciones para emprender. Esto puede ser representativo del contexto ecuatoriano y del déficit de espacios existentes relacionados con ambientes de innovación y emprendimiento.

\section{Limitaciones}

Entre las limitaciones del estudio se encuentra que los datos fueron obtenidos en dos grupos, lo que limita poder extender los resultados a un contexto más amplio. Por tanto, sería de utilidad el poder extender el estudio a otros contextos que permitan verificar el comportamiento específico que están teniendo las variables competencias y condiciones de emprendimiento. Al mismo tiempo, hay una limitación relacionada a la presencia del género, definida por el contexto. Se podría realizar una investigación más amplia, a nivel regional, para poder extender las conclusiones a temas de género que permitan generar políticas de apoyo, así como estrategias de fortalecimiento de los espacios y ambientes de innovación.

\section{Pistas para futuras investigaciones}

El emprendimiento es clave para el desarrollo de los países y, a pesar de que se ha estudiado de acuerdo a la viabilidad económica y necesidad del mismo, es recientemente que se ha incorporado la noción de relación entre competencias y condiciones para emprender, considerado como la tercera ola del emprendimiento (Welter et al., 2019). En este sentido, hay mucho trabajo por hacer para comprender estas relaciones y aspectos como el contexto, los que se han verificado importantes para poder entender el emprendimiento como un proceso multifactorial, como ha sido la propuesta de autores como Vallaster et al. (2019), lo que también constituye uno de los principales hallazgos de esta investigación.

\section{Referencias}

Astudillo, S., Mora, P., \& Pozo, S. (2019). Evaluación de la cátedra de emprendimiento desde su intención emprendedora en una universidad pública en Cuenca. Brazilian Journal of Development.

Bird, B., \& Jelinek, M. (1988). The Operation of Entrepreneurial Intentions. University of Illinois at UrbanaChampaign's Academy for Entrepreneurial Leadership Historical Research Reference in Entrepreneurship. 
Bakir, C., \& Jarvis, D. S. L. (2017). Contextualising the context in policy entrepreneurship and institutional change. Policy and Society, 36(4), 465-478. https://doi.org/10.1080/14494035.2017.1393589

Bjornali, E. S., \& Anne Støren, L. (2012). Examining competence factors that encourage innovative behaviour by European higher education graduate professionals. Journal of Small Business and Enterprise Development, 19(3), 402-423. https://doi.org/10.1108/14626001211250135

Dauvellier, M., Dennehy, J., Duda, A., Ferguson, L., Ferrari, E., Forest, M., Frangoudes, K., Frey, R., Irving, P., Lodovici, M. S., Loi, D., Mcdevitt-pugh, L., Mantouvalou, K., Mergaert, L., Green, S. O. B., Orlando, N., Osch, T. Van, Pesce, F., Pimminger, I., ... Zambelli, M. S. (2016). Gender in entrepreneurship. European Institute for Gender Equality, 22.|

Echeverri, L. V. (2018). Factores que inciden en la intención emprendedora del estudiantado universitario . Educare, 1-19.

Faghih, N. B. (2019). Global Entrepreneurship Capacity and Entrepreneurial Attitude Indexing Based on the Global Entrepreneurship Monitor (GEM). Dataset, 13-55.

Ferreto, E. L. (2018). Capital humano y factores sociológicos como determinantes del emprendimiento. TEC Empresarial , 43-49.

Gaddefors, J., \& Anderson, A. R. (2017). Entrepreneursheep and context: when entrepreneurship is greater than entrepreneurs. International Journal of Entrepreneurial Behaviour and Research, 23(2), 267-278. https://doi.org/10.1108/IJEBR-01-2016-0040

Gallurt Plá, P. (2010). Creación de «spin-offs» en las universidades españolas: un modelo de intenciones. Departamento de Dirección de Empresas

Gamal, D., Salah, T., \& Elrayyes, N. (2011). How to measure organization Innovativeness? Technology Innovation and Entrepreneurship Center, 1-35. http://tiec.com.eg/backend/Reports/MeasuringOrganizationInnovativeness.pdf

Henríquez, M. M. (2010). La creación de empresas en Colombia desde las percepciones femenina y masculina. Economía, Gestión y Desarrollo, 61-77.

Iglesias, P., Jambrino, C., Peñafiel, A., \& Kokash, H. (2016). Impact of entrepreneurship programmes on university students. Education + Training , 209-228.

Ilie, C., \& Cardoza, G. (2018). Emprendimiento y género en América Latina. INCAE Bussines School.

Jones, P., Ratten, V., Klapper, R., \& Fayolle, A. (2019). Entrepreneurial identity and context: Current trends and an agenda for future research. International Journal of Entrepreneurship and Innovation, 20(1), 3-7. https://doi.org/10.1177/1465750319825745

Krauss, C. B. (2018). Modelo predictivo de la intención emprendedora universitaria en Latinoamérica . Journal of technology management \& innovation, 84-93.

Kuratko, D. F., \& Morris, M. H. (2018). Examining the Future Trajectory of Entrepreneurship. Journal of Small Business Management, 56(1), 11-23. https://doi.org/10.1111/jsbm.12364

Lasio, V. O., Caicedo, G., Samaniego, A., Izquierdo, E., Zambrano, J., \& Tenesaca, T. (2017). Global Entrpeneurship Monitor Ecuador 2017. Global Entrpeneurship Monitor, 1-80

Lounsbury, M., Cornelissen, J., Granqvist, N., \& Grodal, S. (2019). Culture, innovation and entrepreneurship. Innovation: Management, Policy and Practice, 21(1), 1-12. https://doi.org/10.1080/14479338.2018.1537716

Majmud, P. (2013). La capacidad empredendora. Obtenido de Red de Emprendimiento: http:/www.redemprendimientoinacap.cl/columna 
Malecki, E. J. (2018). Entrepreneurship and entrepreneurial ecosystems. Geography Compass, 12(3), 1-21. https://doi.org/10.1111/gec3.12359

Marin-Garcia, J. A., Aznar-Mas, L. E., \& Gonzalez-Ladrón-de-Guevara, F. (2011). Innovation Types and Talent Management for Innovation. WPOM-Working Papers on Operations Management, 2(2), 25-31. http://polipapers.upv.es/index.php/WPOM/article/view/926

Mayorga, J. A., Morales, D. C., \& Carvajal, R. P. (2020). Entrepreneurship and gender: a comparative perspective of Latin America and Ecuador. Emprendimiento y Género: Una Perspectiva Comparada de Latinoamérica y Ecuador., 41(3), 144-150.

http://electra.lmu.edu:2048/login?url=https://search.ebscohost.com/login.aspx?direct=true\&db=edb\&A $\mathrm{N}=141766012 \&$ site $=$ eds-live \&scope $=$ site

Meoli, A., Fini, R., Sobrero, M., \& Wiklund, J. (2020). How entrepreneurial intentions influence entrepreneurial career choices: The moderating influence of social context. Journal of Business Venturing, 35(3). https://doi.org/10.1016/j.jbusvent.2019.105982

Nakara, W. A., Messeghem, K., \& Ramaroson, A. (2019). Innovation and entrepreneurship in a context of poverty: a multilevel approach. Small Business Economics. https://doi.org/10.1007/s11187-019-002813

Paturel, R. (1997). Pratique du management stratégique. Les processus de la création d'entreprise. Grenoble:P resses Universitaires de Grenoble.

Pinokvetskaia, I., \& Ginzburg, M. (2018). Women's Entrepreneurship in modern Russia. Revista Espacios, 1-6.

Powell, B. (2013). Dilemmas in entrepreneurship pedagogy. Journal of Entrepreneurship , 99-112.

Ramos, V., Ruiz, A., \& Franco-crespo, A. (2020). The Relationship between Interest and Capacity for Entrepreneurship in University Students. Test Engineering \& Management, May-June, 25803-25815.

Rich, C. R., Vernooij, M., Wadhwa, S. S., Rich, C. R., Vernooij, M., \& Wadhwa, S. S. (2019). Introduction to entrepreneurship. Health Entrepreneurship, April, 1-4. https://doi.org/10.4324/9781315122137-1

Roegiers, X. (2000). Saberes, capacidades y competencias en la escuela: una búsqueda. Innovación educativa, 103-119.

Sanz-Valle, R., Naranjo-Valencia, J. C., Jiménez-Jiménez, D., \& Perez-Caballero, L. (2011). Linking organizational learning with technical innovation and organizational culture. Journal of Knowledge Management, 15(6), 997-1015. https://doi.org/10.1108/13673271111179334

Sarfaraz, L., Faghih, N., \& Majd, A. A. (2014). The relationship between women entrepreneurship and gender equality. Journal of Global Entrepreneurship Research, 2(6), 2-11. https://doi.org/10.1016/j.cap.2005.04.024

Schmutzler, J., Andonova, V., \& Diaz-Serrano, L. (2019). How Context Shapes Entrepreneurial Self-Efficacy as a Driver of Entrepreneurial Intentions: A Multilevel Approach. Entrepreneurship: Theory and Practice, 43(5), 880-920. https://doi.org/10.1177/1042258717753142

Sullivan, D. M., \& Meek, W. R. (2012). Gender and entrepreneurship: A review and process model. In Journal of Managerial Psychology (Vol. 27, Issue 5). https://doi.org/10.1108/02683941211235373

Vallaster, C., Kraus, S., Merigó Lindahl, J. M., \& Nielsen, A. (2019). Ethics and entrepreneurship: A bibliometric study and literature review. Journal of Business Research, 99(February), 226-237. https://doi.org/10.1016/j.jbusres.2019.02.050

Villalobos, G. V. (2018). Lean start-up as strategy for the development and management od dynamic entrepreneurships. Dimensión Empresarial, 193-208. 
Vuorio, A. M., Puumalainen, K., \& Fellnhofer, K. (2018). Drivers of entrepreneurial intentions in sustainable entrepreneurship. International Journal of Entrepreneurial Behaviour and Research, 24(2), 359-381. https://doi.org/10.1108/IJEBR-03-2016-0097

Ward, A., Hernández-Sánchez, B. R., \& Sánchez-García, J. C. (2019). Entrepreneurial Potential and Gender Effects: The Role of Personality Traits in University Students' Entrepreneurial Intentions. Frontiers in Psychology, 10(December), 1-18. https://doi.org/10.3389/fpsyg.2019.02700

Welter, F., Baker, T., Audretsch, D. B., \& Gartner, W. B. (2017). Everyday Entrepreneurship_A Call for Entrepreneurship Research to Embrace Entrepreneurial Diversity. Entrepreneurship: Theory and Practice, 41(3), 311-321. https://doi.org/10.1111/etap.12258

Welter, F., Baker, T., \& Wirsching, K. (2019). Three waves and counting: the rising tide of contextualization in entrepreneurship research. Small Business Economics, 52(2), 319-330. https://doi.org/10.1007/s11187018-0094-5

Yépez-Mora, M., Frías-Campos, E., \& Asunción-Parrales, R. (2019). Innovación y emprendimiento como nuevo reto del siglo XXI en la sociedad. 